\title{
Impact of olaparib use on BRCA-mutated cancer patients in Qatar: Medication use evaluation
}

\author{
Hebatallah Afifi, Arwa Sahal, Amaal Gulied, Farah Jibril, Nabil Omar, Aya Alasmar, Anas \\ Hamad, Shereen Elazzazy* \\ Pharmacy Department- National Center for Cancer Care and Research, Hamad Medical Corporation, Doha, Qatar
}

\section{Background}

Olaparib is the first approved PARPs inhibitor for ovarian and breast cancer. Bone marrow suppression, gastrointestinal side effects, and fatigue are the most common toxicities with olaparib. PARP inhibitors are costly drugs; costbenefit ratio should be considered.

Primary Objective: evaluating the response on olaparib. Secondary Objectives: assessing our practice and adherence to the international guidelines, detecting medication-related problems, and enhance to incident reporting.

\section{Methodology}

Retrospective analysis of data from all patients who received olaparib (for different indications) as over the period of 2 years.

\section{Results}

Olaparib was used for 9 patients as an advanced line of therapy. BRCA-mutated ovarian and breast cancer presented $55 \%$ and $22 \%$, respectively, while other cancer types represented $23 \%$. The median duration of treatment was 3 months. The overall discontinuation due to lack of benefit was $88 \%$, $60 \%$ in ovarian and $100 \%$ in other cancer types, while, $12 \%$ of discontinuations were due to side effects. Around $63 \%$ of patients had one or more level of dose reduction due to toxicity. The most common side effects were Gl, bone marrow suppression, hepatotoxicity, and hyperglycemia. Only $25 \%$ of cases were deceased at the time of data evaluation due to different reasons.

\section{Conclusion}

Olaparib is a well-tolerated drug. However, discontinuation of the drug due to disease progression was relatively high. Adherence to approved indications should be reevaluated to minimize treatment discontinuation due to the lack of benefit, and decrease unnecessary cost. Reporting of rare side effects is adequate in NCCCR. However, there is still a room for improvement.

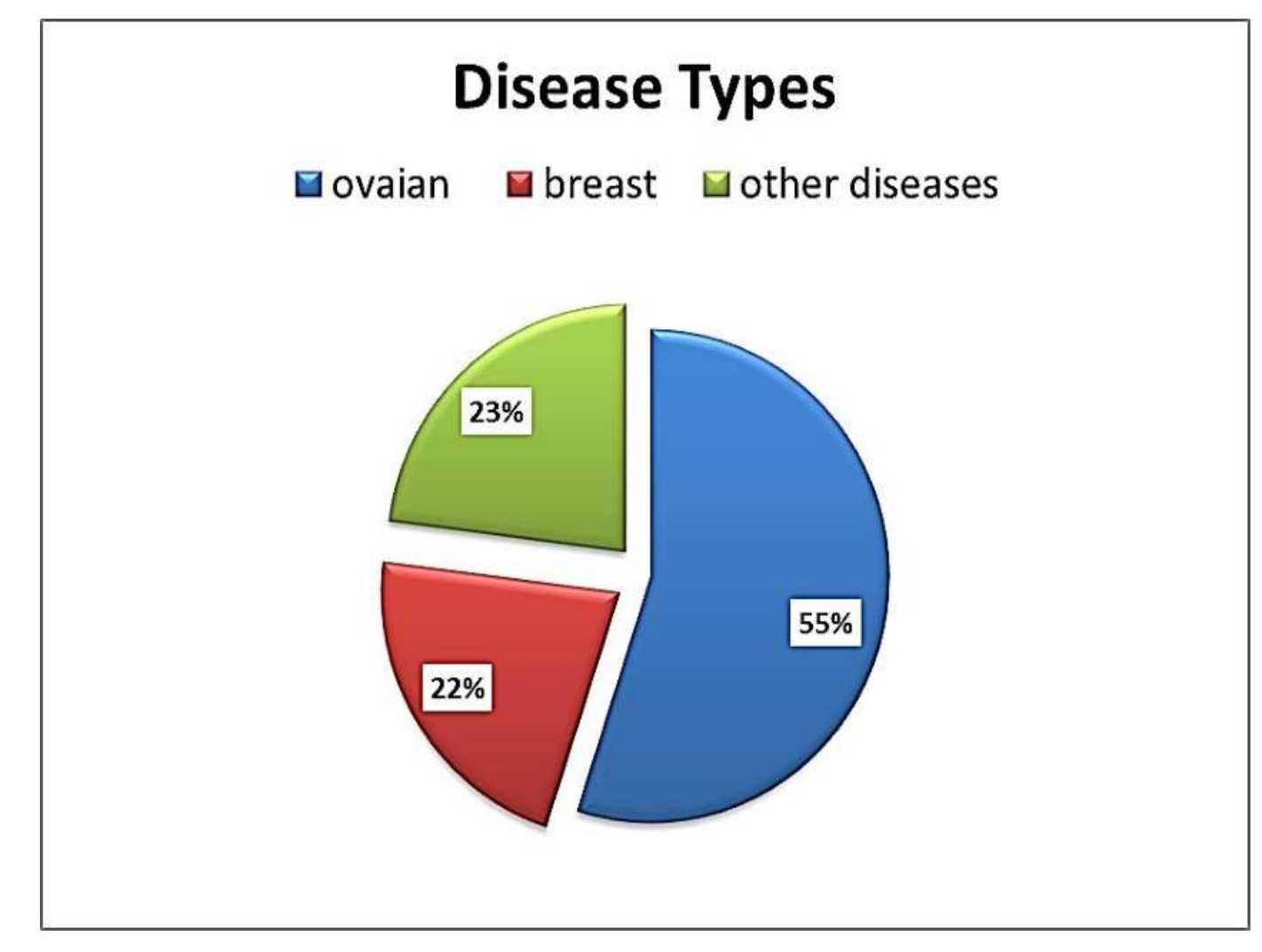

\section{Acknowledgement}

To Medical Research Center (MRC) of Hamad Medical Corporation (HMC) for funding this study

\section{References}

1. Audeh MW, Carmichael J, Penson RT, et al. Oral poly(ADP-ribose) polymerase inhibitor olaparib in patients with BRCA1 or BRCA2 mutations and recurrent ovarian cancer: a proof-of-concept trial. Lancet. 2010;376(9737):245-251.[PubMed 20609468]

2. Domchek SM, Aghajanian C, Shapira-Frommer $R$, et al. Efficacy and safety of olaparib monotherapy in germline BRCA1/2 mutation carriers with advanced ovarian cancer and three or more lines of prior therapy. Gynecol Oncol. 2016;140(2):199-203.[PubMed 26723501]

3. Robson M, Im SA, Senkus E, et al. Olaparib for Metastatic Breast Cancer in Patients with a Germline BRCA Mutation. N Engl J Med. 2017;377(6):523-533. doi: 10.1056/NEJMoa1706450.[PubMed 28578601] 